\title{
Inherent Photoluminescence Properties of Poly(Propyl Ether Imine)
}

\section{Dendrimers}

\author{
G. Jayamurugan, C. P. Umesh and N. Jayaraman* \\ Department of Organic Chemistry, Indian Institute of Science, Bangalore 560 012, India \\ jayaraman@orgchem.iisc.ernet.in
}

\section{Supplementary Information}

Methods: Absorption and fluorescence spectra were recorded using quartz cell with a path length of $1 \mathrm{~cm}$. Absorption spectra were recorded on a Perkin-Elmer lambda 35 spectrophotometer at a scan rate of $120 \mathrm{~nm} / \min$ and a width of $2 \mathrm{~nm}$. The absorption

data were blank corrected with absorption of the solvent. Fluorescence spectra were recorded on a Shimadzu RF-540 spectrofluorophotometer. Distilled deionized water (Millipore filtration system) was used for the experiments. Fluorescence lifetime spectrophotometer (Model 5000 U, IBH, UK) with a Time Correlated Single Photon Counting technique (TCSPC) and microchannel plate photomultiplier tube (MCP-PMT) as detector was used to conduct the lifetime measurements. A picosecond laser was used as the excitation source. The excitation wavelength is $267 \mathrm{~nm}$, which was produced by using third harmonic laser and the emission decay was monitored at the wavelength of $390 \mathrm{~nm}$. Fluorescence quenching studies were performed using aqueous G5 (0.1 mM) solution, titrated with a sample of the quenchers in water. 


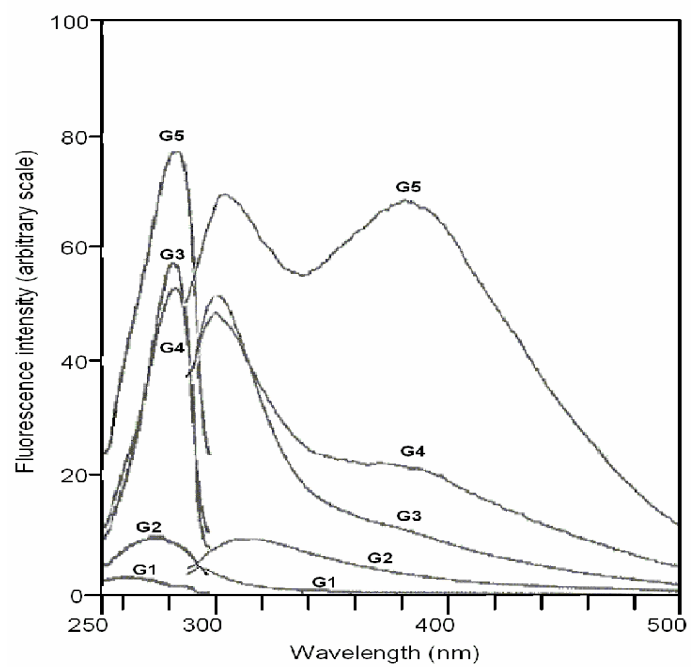

Figure 1. Emission and excitation spectra of methanolic solutions of PETIM dendrimer generations at $\lambda_{\mathrm{ex}}=273 \mathrm{~nm}$. [G1]-[G3] $=0.5 \mathrm{mM} ;[\mathbf{G 4}]=0.25 \mathrm{mM} ;[\mathbf{G 5}]=0.125 \mathrm{mM}$.

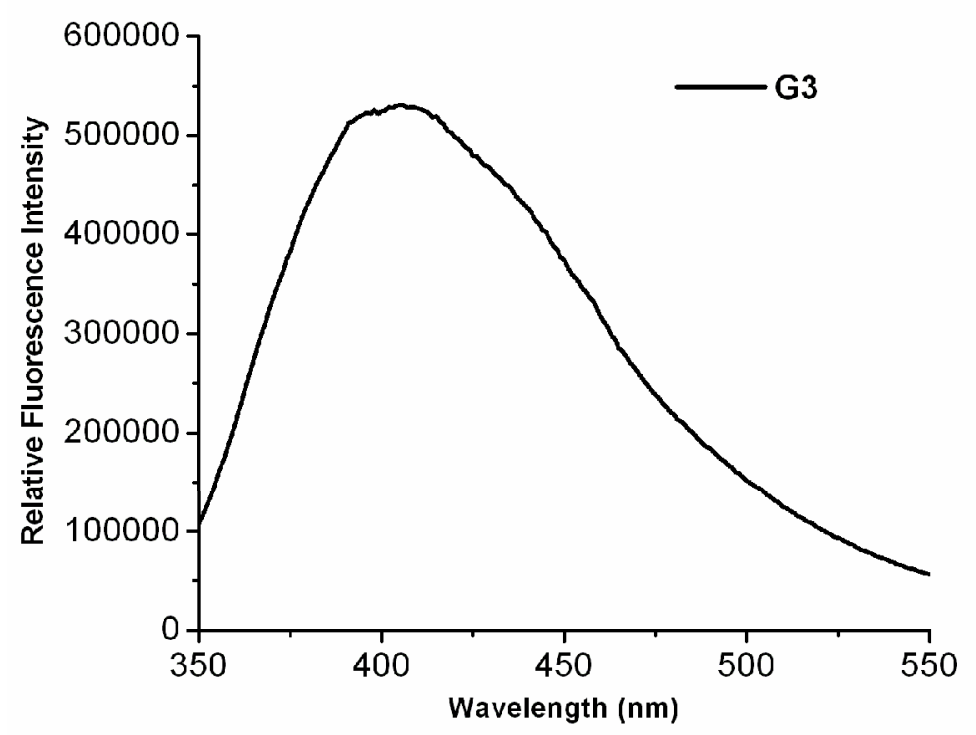

Figure 2. Thin film emission spectrum of $\mathbf{G 3}\left(\lambda_{\mathrm{ex}}=330 \mathrm{~nm}\right)$. 


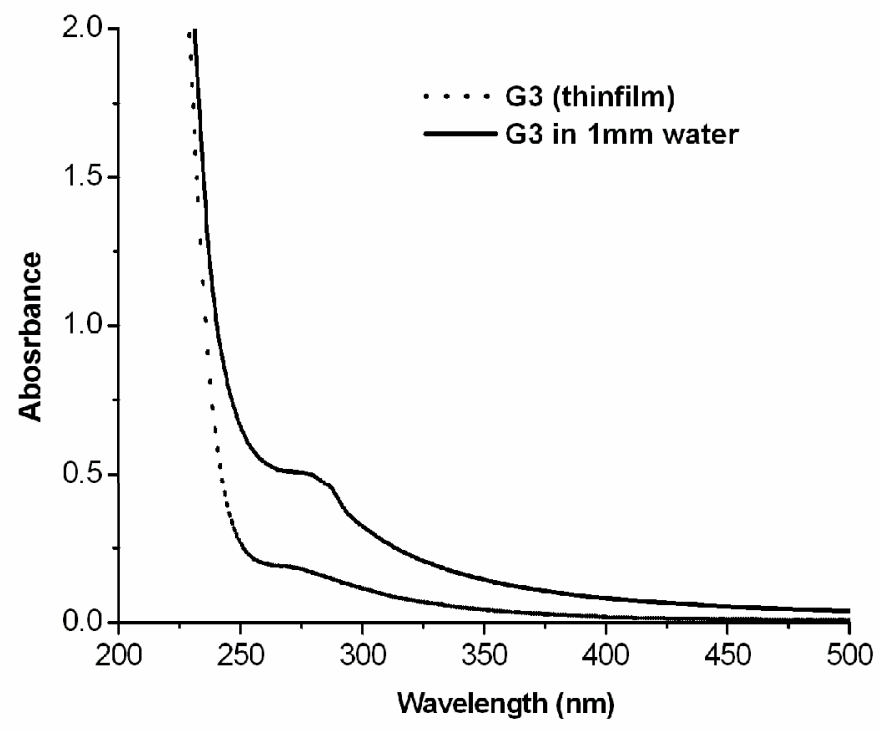

Figure 3. Thin film and solution absorption spectrum.

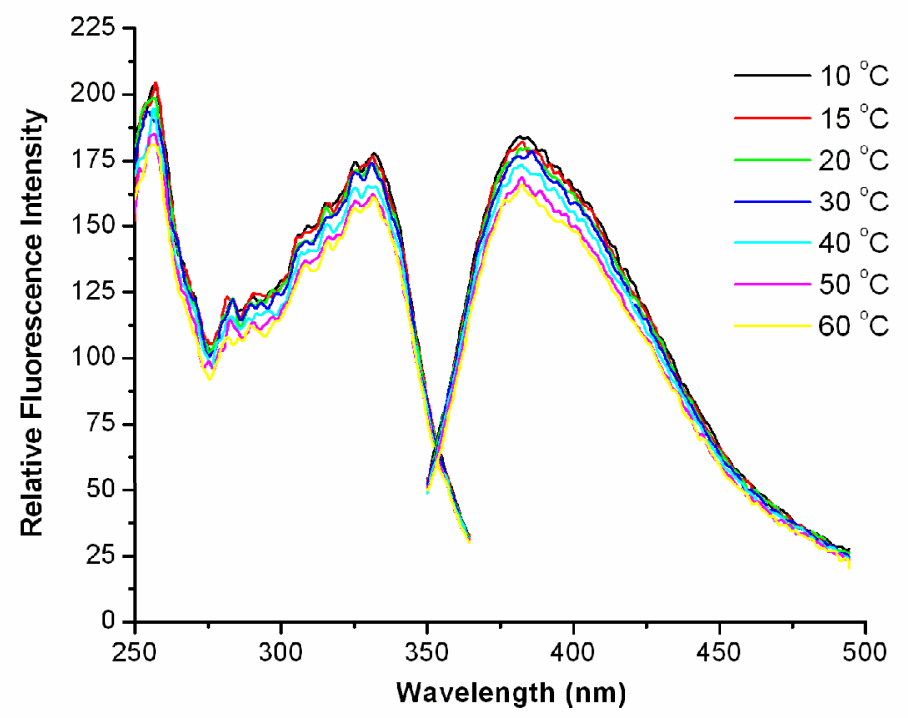

Figure 4. Variable temperature excitation and emission spectra of an aqueous solution of the $\mathbf{G 4}$ dendrimer (0.5 $\mathrm{mM})$. 


\section{Day 0}

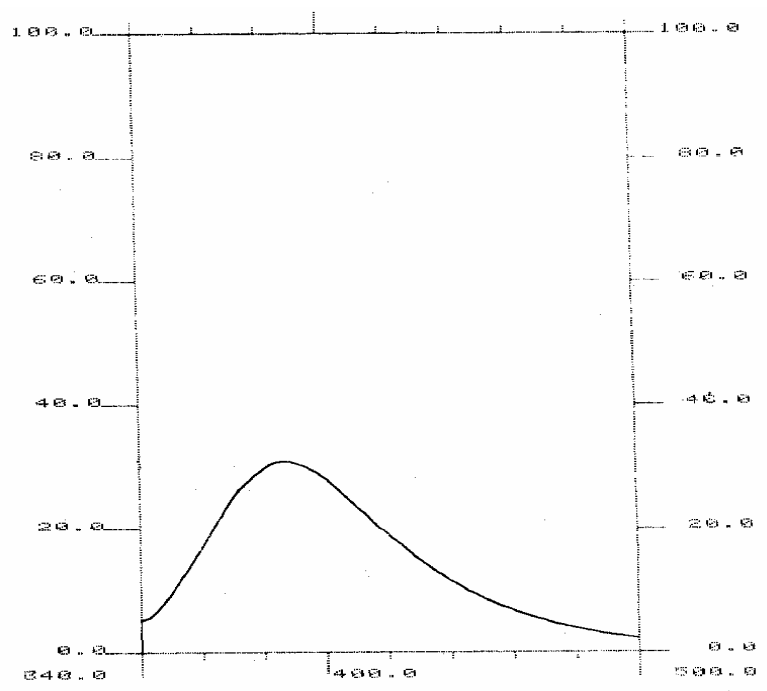

Day 24

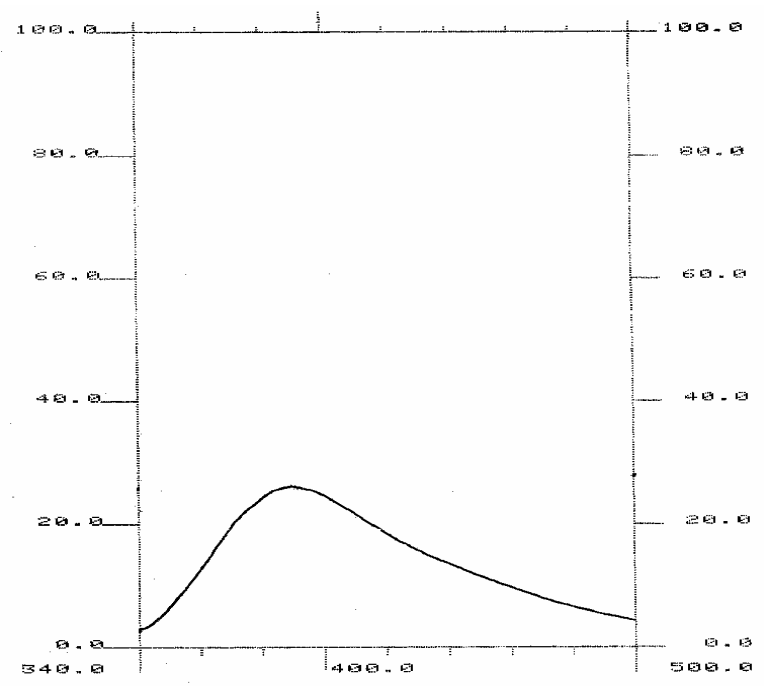

$\underline{\text { Day } 45}$

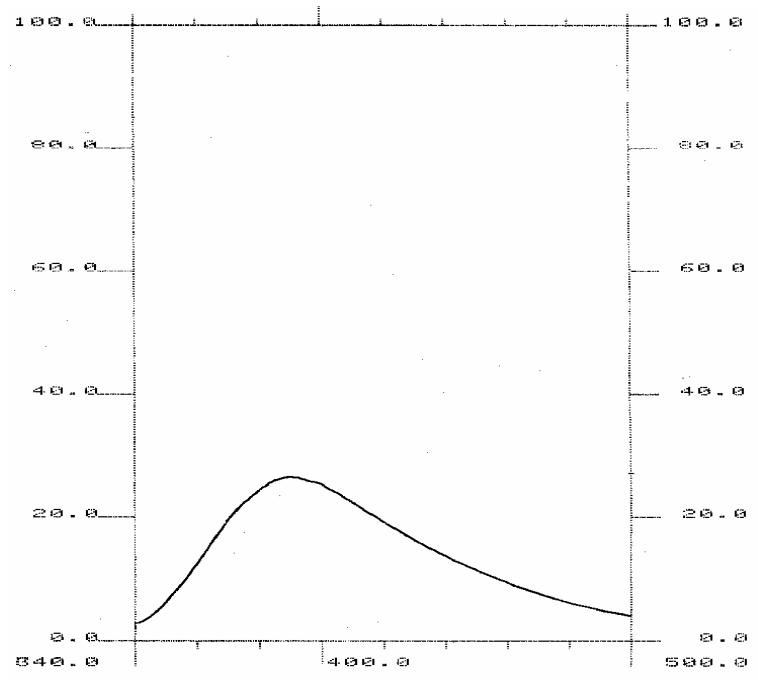

$\underline{\text { Relative fluorescence intensity } v s \text { number of days }}$

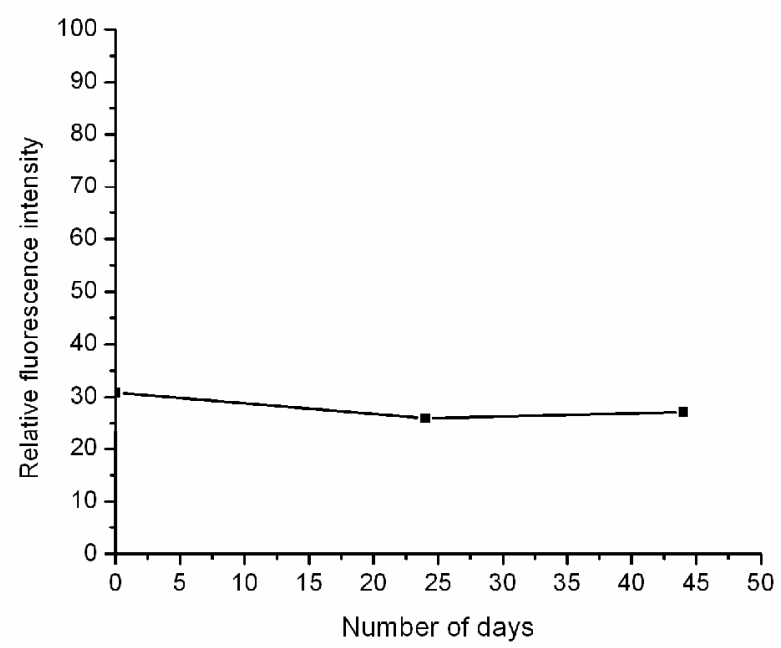

Figure 5. Emission spectra of an aqueous solution of $\mathbf{G 4}$ dendrimer $(0.7 \mathrm{mM})$, purged with nitrogen and kept at $50{ }^{\circ} \mathrm{C}$, measured at different intervals. 


\section{Day 0}

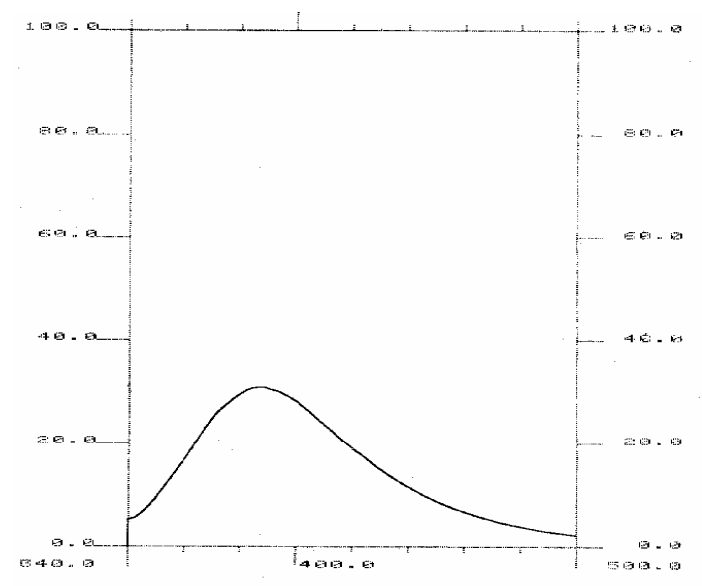

\section{Day 16}

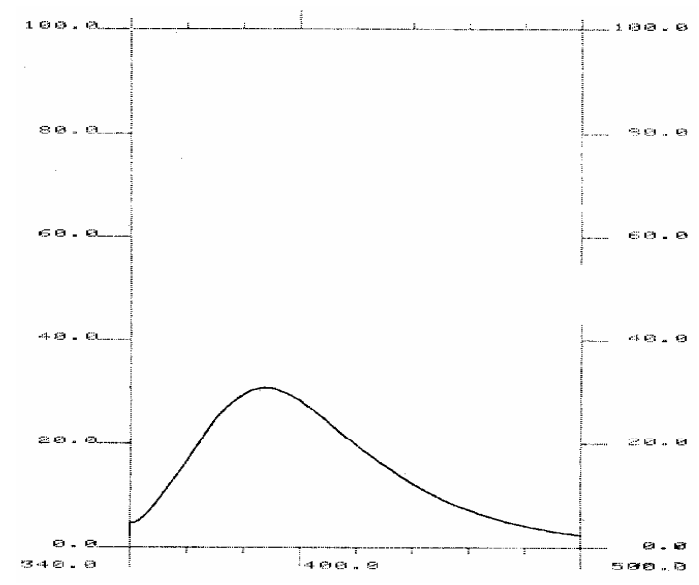

\section{$\underline{\text { Day } 45}$}

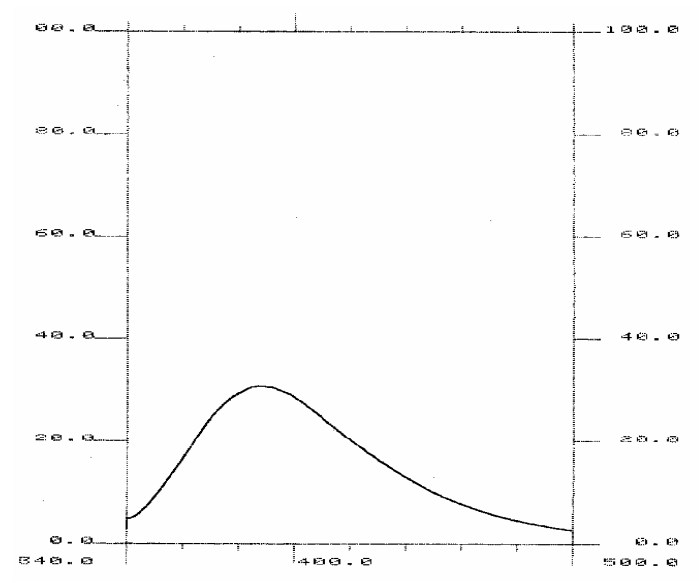

\section{Day 5}

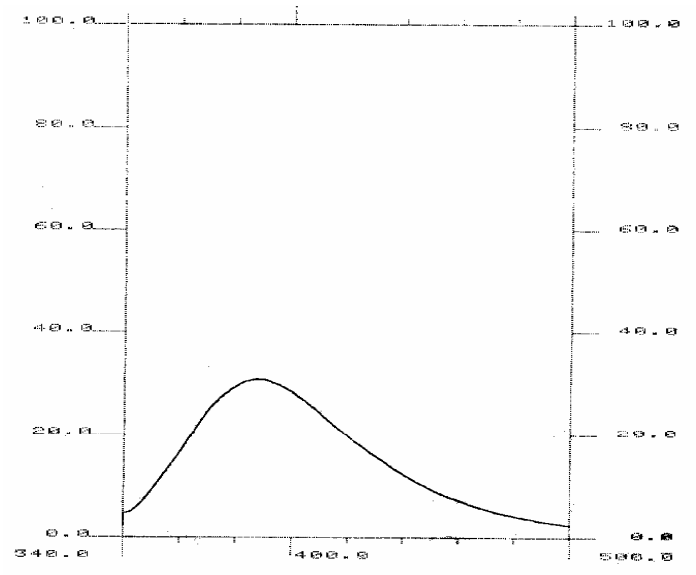

Day 24

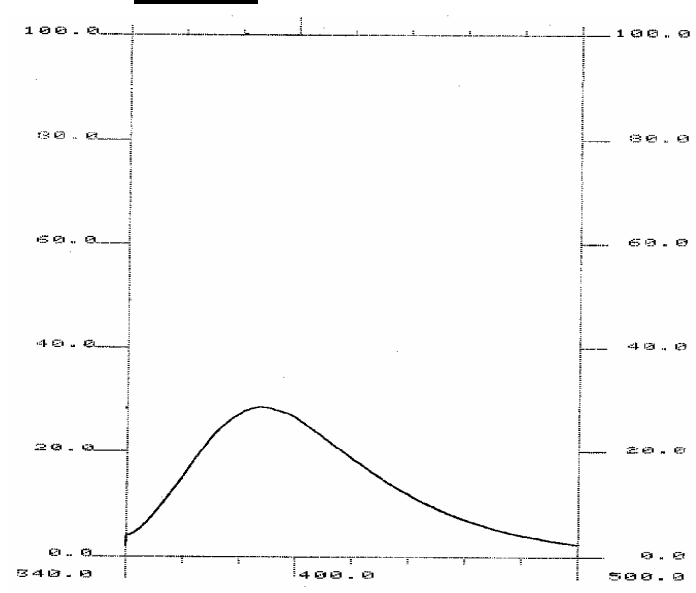

Relative fluorescence intensity vs number of days

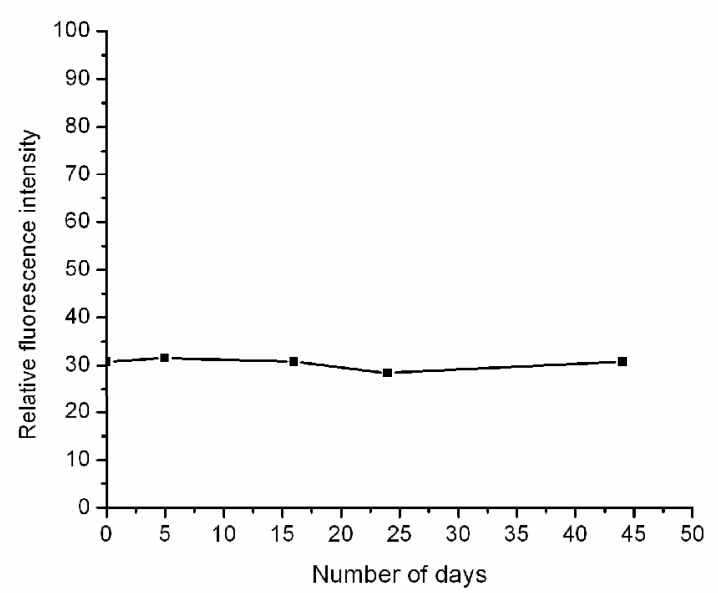

Figure 6. Emission spectra of an aqueous solution of $\mathbf{G 4}$ dendrimer $(0.7 \mathrm{mM})$, purged with air and kept at $0{ }^{\circ} \mathrm{C}$, measured at different intervals. 


\section{Day 0}

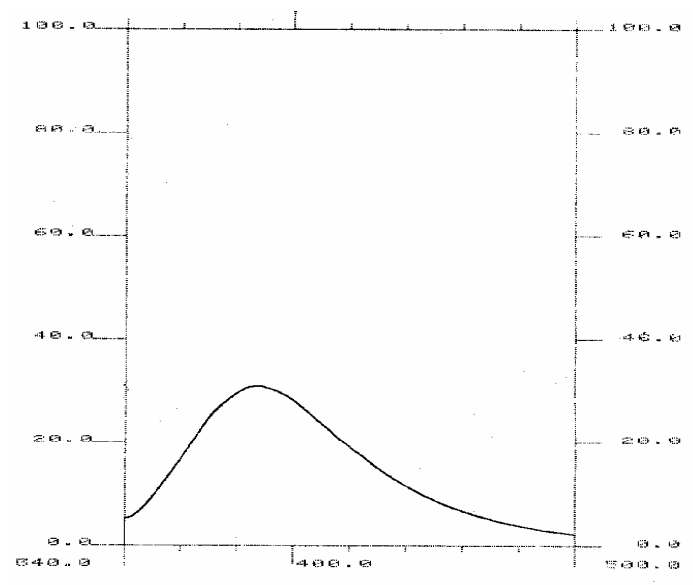

\section{Day 16}

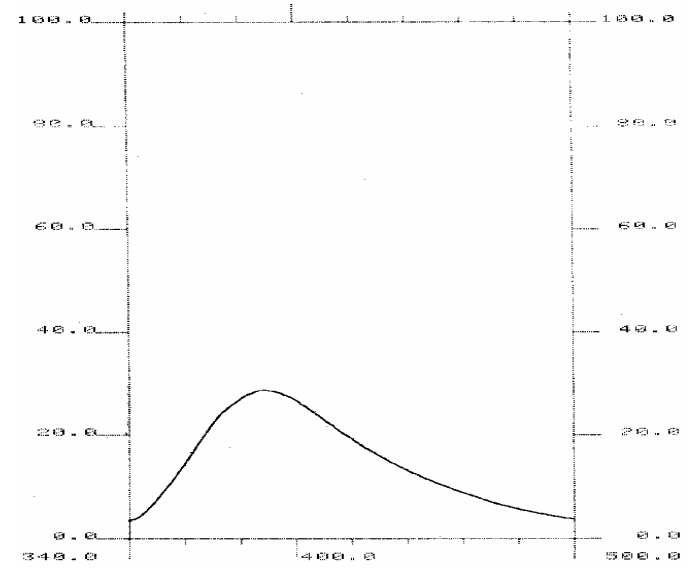

\section{Day 45}

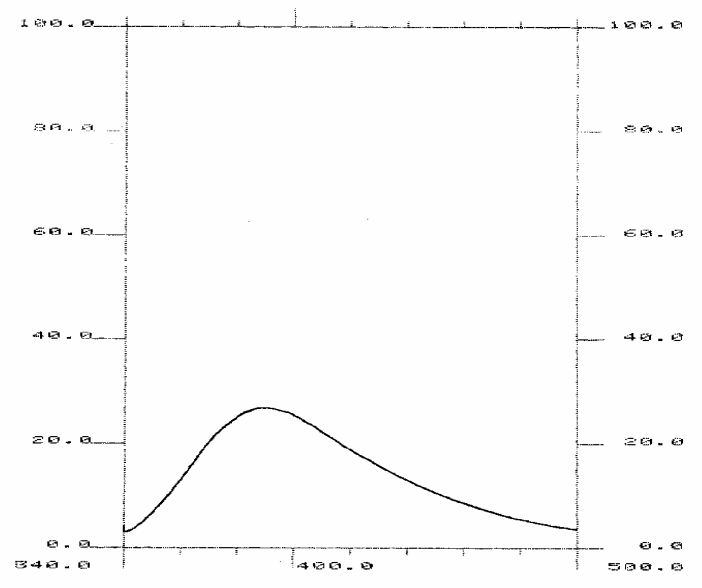

Day 5

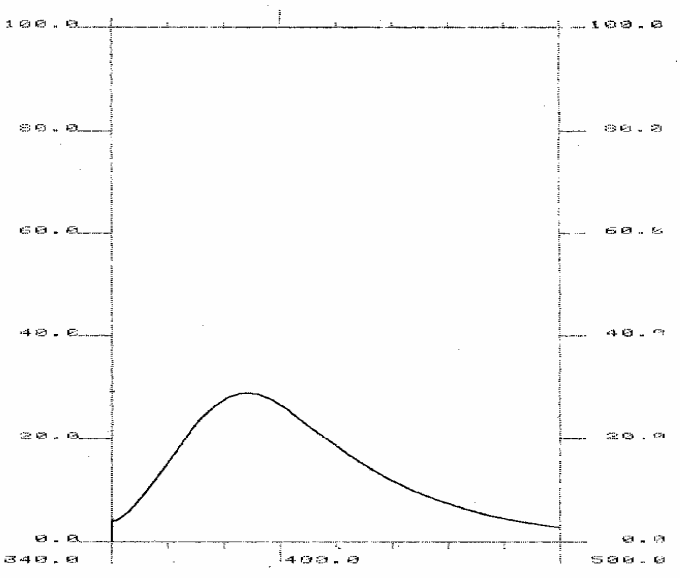

Day 24

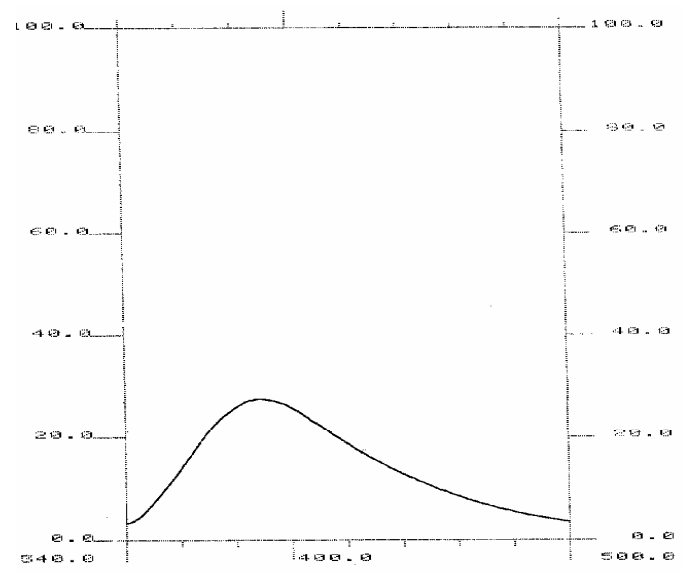

Relative fluorescence intensity vs number of days

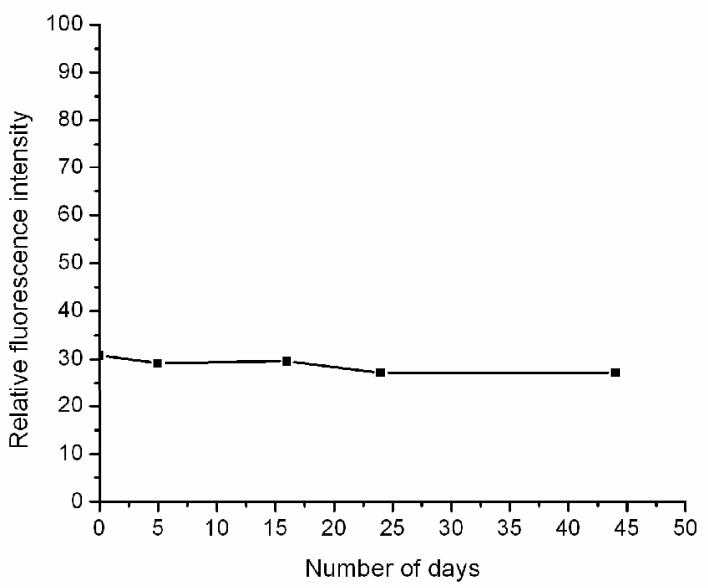

Figure 7. Emission spectra of an aqueous solution of $\mathbf{G 4}$ dendrimer $(0.7 \mathrm{mM})$, purged with air and kept at $50{ }^{\circ} \mathrm{C}$, measured at different intervals. 


\section{Day 0}

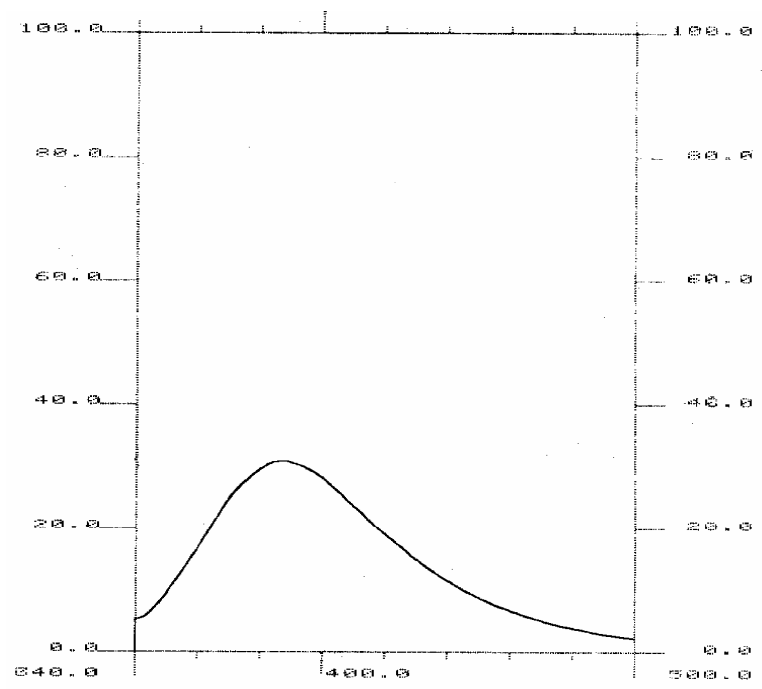

Day 24

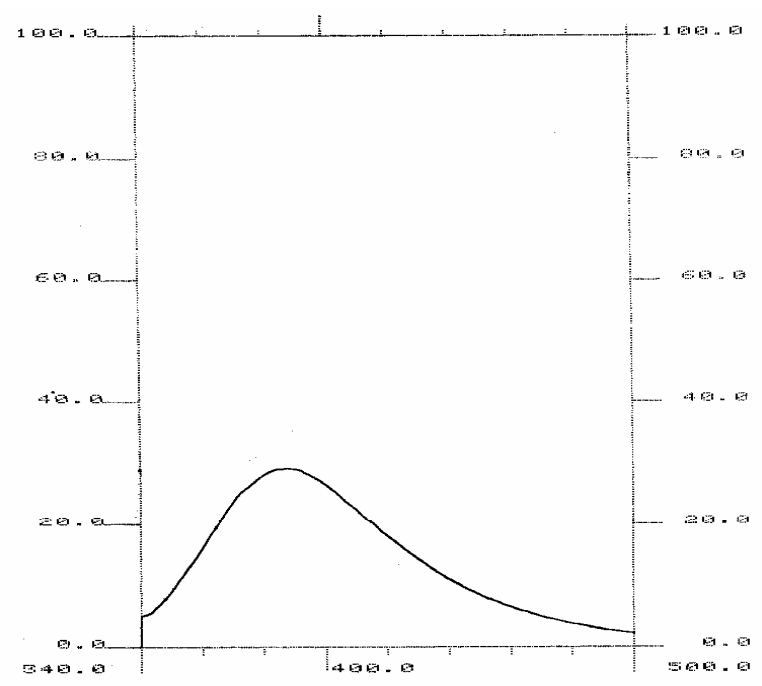

\section{Day 12}

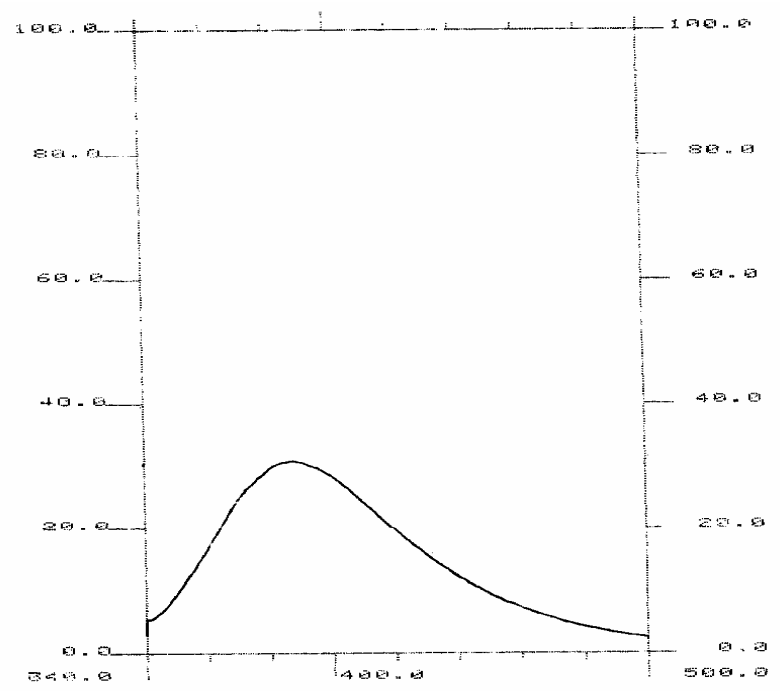

$\underline{\text { Relative fluorescence intensity vs number of days }}$

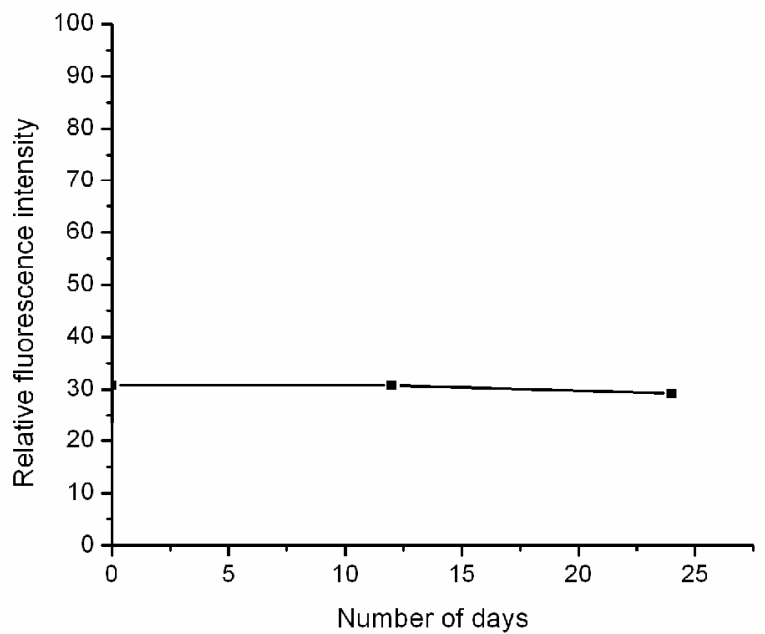

Figure 8. Emission spectra of an aqueous solution of $\mathbf{G 4}$ dendrimer $(0.7 \mathrm{mM})$, purged with air and kept at room temperature, measured at different intervals. 


\section{Lifetime Measurements}

Fluorescence lifetime distributions for PETIM dendrimers excited at $330 \mathrm{~nm}$, using a nanosecond flash lamp.

\begin{tabular}{cccccc}
\hline Sample & $\lambda_{\text {ex }}$ & $\lambda_{\mathrm{em}}$ & $\mathrm{T} 1(\alpha 1)$ & $\mathrm{T} 2(\alpha 2)$ & $\mathrm{T} 3(\alpha 3)$ \\
\hline $\mathrm{G} 4 \mathrm{H}_{2} \mathrm{O}$ & $330 \mathrm{~nm}$ & $390 \mathrm{~nm}$ & $1.90 \mathrm{~ns}(0.37)$ & $0.25 \mathrm{~ns}(0.17)$ & $6.98 \mathrm{~ns}(0.45)$ \\
\hline
\end{tabular}

Fluorescence lifetime spectrophotometer (model 5000 F) with a Time Correlated Single

Photon Counting technique (TCSPC) was used to conduct the life time measurements. 